\title{
A Review of Relationships Between Attention and Emotion
}

\author{
Lokyin Chan ${ }^{1, *,}$, Yutong Xie ${ }^{2, *, \dagger}$, Xueyu Zhao ${ }^{3, \dagger}$

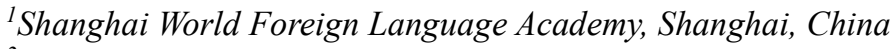 \\ ${ }^{2}$ Beijing Beanstalk International Bilingual School, Beijing, China \\ ${ }^{3}$ Beijing National Day School, Beijing, China \\ *Corresponding author.Email: 'lokyin_chan@stu.swfla.org, ${ }^{2} Y u t o n g X i e 2022 @ b i b s . c o m . c n$ \\ These authors contributed equally.
}

\begin{abstract}
Attention is crucially important in deciding human activities, such as rewards and emotions. However, attention itself does not dominate the whole authority. Well engaged with emotions, attention can produce a completely different result. Over recent decades, the relationship between emotion and attention has been well investigated. This paper will investigate and analyze different studies understanding of the interaction between attention and emotion. First, theories considering the relationship between attention and emotion will be introduced. This would provide insight into how proven theories view the relationship between the two subjects. Next, several studies, together with their methodology and results, would be presented. The studies commendably proved hypothetical guesses about how attention and emotion may affect each other.
\end{abstract}

Keywords: Attention, Emotion, biased competition, Event-related brain potentials (ERP).

\section{INTRODUCTION}

When students realize that their exam dates or their deadline is approaching, they may start to feel anxious and frustrated, making them either more concentrated on their studies or deciding to give up. When people are listening to a speech, lecturers with a fixed tone of voice usually make the audience drowsy, while those who are full of emotion tend to attract spectators' attention. These examples from daily life demonstrate the underlying impact of emotions on human attention. Recent decades have witnessed the progress of researches on the relationship between attention and emotion. Before going further into the relationship between the two, it is necessary to define them clearly.

In order to further the research on the interaction between attention and emotion, it is crucial to have a clear perspective on the definition of these two concepts. Up until now, there has not been a specific and precise definition that could be given to attention. It's undeniable that under different situations, the definition of attention could be different. Attention is such a great academic area that could not be explicitly explained in a simple definition. However, different authors have given different attitudes toward the definition of attention. (i)
Attention is a psychological activity that describes various phenomena [1]. (ii) Attention is a mechanism that monitors which brain processes are allowed to enter access awareness and which cannot $[2,3]$. (iii) Attention is a burgeoning phenomenon coming from re-entry and slow competitive interactions $[4,5]$. Again, the definition given to attention is different in different situations due to various types of attention. As with discussion of emotion, selective attention would be mainly considered, and focus mainly on visual attentions. First, in foveal short-sustained, long-sustained, and vigilance attention tasks. Second, in covert attention task, subjects attend peripheral stimulus without moving eyes. Third, in divided attention task, subjects divide their attention between various stimuli. Fourth, in selective attention task, subjects focus on the target and ignore disturbance terms [6-8]. Fifth, in switching attention task, subjects switch attention to other tasks by disintegrating attention from the first and engaging to the second.

As for emotion, it is a complex behavioural phenomenon. Emotions include anger, disgust, fear, joy, sadness, and surprise [9]. Having a strong sense of emotions represents a man with high level of sympathy. Sometimes positive and negative emotions are the key theoretical structures. Emotions have been studied in 
several scientific disciplines, such as biology, psychology, etc. As a result, there are different views on emotions. For example, in psychology, letting someone have too many emotions with them [overwhelming happiness, overwhelming sadness] is a mental issue, however, if one has a lot of emotions when communicating with others, that is actually, a pretty good thing [10].

The following review focuses on the possible ways in which emotion influences attention. Overall, people, objects, and events that arouse our emotions can attract our attention. It is a fact that when people are performing tasks in a strong emotional state, their limited attention resource would be divided, which makes them soak in that specific emotion rather than focusing on the task. First, an overview of the theories regarding the interaction between emotion and attention. Next, some experimental evidences supporting the relations between emotion and attention will be reviewed.

\section{THEORIES}

\subsection{Biased Competition Theory}

Biased competition is a theory of attention proposed, stating that stimulus will compete for representation [1113]. Humans only own a processing system with a limited-capacity though need to deal with overloading information, including internal and external stimulus. Competition between these pieces of information is crucial to ensure no supercharging of information occurs. Since top-down and bottom-up factors are able to influence the activation of representations, it is therefore called "biased" in terms of the competition. Undeniably, information must be selected, remaining the important part, and rejecting the remainder. The brain must then develop a system or a measure to select the information based on a theory. While considering attention together with emotion, it's crucial to notice that emotional materials may increase salience, and lead to attentional prioritization of these materials (bottom-up factors). Considering top-down factors, they could also bring up similar prioritization as bottom-up factors. Examples such as prior perceptions and past experiences could bring about similar biased competitions [14]. In general, information provided with any enhancing effect may gain priority in the selective attention, and be best understood by the system. Remaining the left to be selectively "not noticed". However, noticing competition is often used within two or more subjects, and only a single emotional stimulus could not be accounted under selective attention. Instead, stimulus should appear simultaneously, and at least two should be accounted.

\subsection{Cognitive Motivated Analysis}

Cognitive motivated analysis is a model that focuses on the effects of a state of anxiety and trait anxiety on the cognitive processing of threat. Anxiety is conceptualized as an aversive motivational state [15]. The cognitive motivated analysis mainly has two cognitive structures. One is called VES which stands for valence evaluation system and GES which stands for goal engagement system. GES has a mode called "safety mode", this mode first thinks about the positive simulations, and will consider or even ignore the negative stimulations. When not considering the basic stimulations, VES will change due to the state of anxiety and the traits of anxiety. The VES is really sensitive with people that have higher levels of anxiety compared with people that have lower levels of anxiety. That results in individuals with higher levels of anxiety were rated as "highly threatening", while individuals with low levels of anxiety were not. The attention will be separated differently due to the level of the threat. Therefore, unlike the other models that focus on how does the system reacts to the threat, this model focus on what constitutes a threat instead $[15,16]$.

\subsection{Feature Integration Theory}

The Feature Integration Theory proposed by Anne Treisman is a milestone in cognitive psychology [17], which illustrates the way perceptual features, such as colour and depth, go through a process of being automatically processed before selective attention happens. Attention is considered the process of representing a relatively complicated stimulus by fusing individual characteristics together [18].

Feature Integration Theory successfully integrated cognitive psychology, neuropsychology, and psychophysics. It was when the cognitive revolution took place during the 1950s researchers started to study the nature of mental processes (such as memory and attention). According to the findings in cognitive psychology, an "internal mechanism" such as attention can be used to explain the process of stimuli being selected for processing. Treisman's work played an important role in illustrating the traits of attentional filter, which is one of the ideas that inspired the feature integration theory. Another is that different processing steps are involved in the processing of stimulus. Additionally, in psychophysics, feature integration theory has succeeded in no small measure in providing a framework for and governing a number of disparate theories [17].

When being applied to a visual search experiment, several stimuli will be presented to study participants' reaction time towards those differing ones by asking subjects to point out them as soon as they detect them. It turns out that participants' performances are always excellent when there is only a single feature is presented, 
regardless of the interference of distracters. However, if complex features are appearing simultaneously, participants tend to perform increasingly erroneously with the increase of the number of stimuli. Normally, reaction times are negatively correlated with the number of distracters [18].

\section{STUDIES}

\subsection{Approach Motivated Positive Affect Reduces Breadth of Attention}

Theories propose that more attentional focus after viewing low approach motivating positive stimuli than after viewing high approach motivating positive stimuli. This is because participants tend not to focus on irrelevant objects when they approach desired objects. Several studies were conducted that proofs the hypothesis.

With preceding overviews, Harmon-Jones and Gable invited 39 participants to the experiment. Participants were given the same piece of video clip to watch, aiming to make them feel neutral before the formal experiment after they were given instructions for the local-global task. Next, participants were randomly assigned to two different groups: either watching a video clip with highapproach motivating positive stimuli, which is delicious desserts, or low-approach-motivating positive stimuli, which depicting humorous situation cats. After the experiment, they will do Kimchi and Palmer's 24-item local-global visual-processing task [19]. This task results that various elements were processed as texture and forms, and, global and local elements normally were processed separately [19]. The experiment allows testing the breadth of attention [20]. A higher score in the task indicates participants show more global focus, and vice versa [21]. The results show that participants were more global after a cat clip compared with a dessert clip. This result indicates that attentional focus would be broader when experiencing low-approach motivating positive stimuli than that of high-approach motivating positive stimuli.

Considering the above experiment does not include the neutrality effect, Gable and Harmon-Jones conducted another study that includes neutral trails. Participants were given six neutral trails (photographs of rocks) followed by 64 experimental trails (graphs of rocks or deserts). Throughout the exhibiting of the trails, the Navon's letter task was engaged to test the attentional breadth [22]. Task, for example, a letter J composed of many closely packed I's. Participants were required to respond whether they see the global or local targets as fast as possible. Ideally, responding to the larger letters indicates a global focus, and oppositely, responding to smaller letters indicates a local focus. [23]. Together with the previous study, they both concluded that a high- approach positive effect could reduce the breadth of attention focus [24].

\subsection{Event-related Brain Potentials}

Event-related brain potentials (ERP) is derived from the electroencephalogram (EEG), which is useful to assess the correlation between attention and emotion at every different level of processing and of great advantages [25].

ERP opens up new possibilities for the brain to process information, allowing emotional cues to be carefully studied. This approach has played an important role in operating selective processing activated by reflexive or explicit attention. To be specific, the processes of perceptual encoding, working memory, and motor preparation are considered to be demonstrated by ERP studies that are applied to experiments such as "the attentional blink, refractory period, visual search, and spatial cuing paradigms" [26]. Three areas have attracted particular attention of researchers among the valuable information offering the emotional perspective of selective attention that provided by ERPs, namely pinpointing particular processing stages at which emotional signals are selectively processed, electing whether the distinctive emotional cues processing has an impact on processing phases, and collating the ERP version of attentional control [25].

ERP measures alone are helpful, however, in revealing the magnitude of attentional resources that are being recruited and the duration that these resources are being utilized to process a particular stimulus or set of stimuli [27]. For example, two ERP studies that showed cocaine and neutral pictures to recent abstinent cocainedependent individuals found greater ERP was positively linked with cocaine craving, especially for the with the late positive potential. ERP component that is up to $1500 \mathrm{~ms}$ in those studies is also thought to reflect the motivational salience of a stimulus. The results suggest cocaine users might use cocaine for an extended duration of time [27]. In the study above, abstinent cocainedependent patients exhibited larger LPP amplitudes in central brain regions in response to heroin-related images, activation that was correlated with higher post experiment negative reinforcement craving ratings [27].

The ERPs were adopted to study facilitated emotional stimulus so as to prove that labelling emotional stimuli in an early and automatic way may help select significant stimuli to prioritize. The examinators invited 15 participants from the University of Greifswald. Three categories of stimuli, namely "pleasant", "neutral", and "unpleasant", were presented. Task-related stimuli were $\mathrm{red} /$ white or yellow/white images with a black or white rectangle in the center. Participants were asked to count those images with black or white rectangle respectively. A 129-lead geodesic sensor net was used to measure the 
brain and ocular scalp potentials. The statistical control of artifact was the basis of data editing. The results of the study turned out to be consistent with the previous hypothesis. Participants' performances in the target discrimination task were good, nine of them counted the images entirely right and the remaining of them only made one or two mistakes. The results of event-related potentials also indicate that participants were focused on the explicit task. Additionally, even though participants carried out an explicit non-emotional task, emotionsignificant stimuli appeared to be selectively processed as the EPN amplitudes increased when pleasant and unpleasant stimuli occurred compared to neutral ones. Further analyses also confirm the hypothesis that stimuli with higher evolutionary significance can evoke greater EPN amplitudes, for example, pictures of threat gave rise to larger EPN amplitudes than did pictures that make less evolutionary sense [28].

In brief, ERP is a type of measurement that has unique advantages and high temporal resolution, which can be adopted to study the relation

\section{CONCLUSION}

The review includes various definitions given to attention and emotion, and how these terms differ in different contexts. As the paper aims to discuss and analyze the interactions between emotion and attention, the paper presents several theories upon their relationships. Despite the pre-existing theories, the paper also presents studies that in different perspectives and explores the relationship between attention and emotion. One of the studies discovers how Approach motivated positive effect could work to reduce the breadth of attention. As a conclusion, this paper could well benefit researchers who aim to discover the interactions between attention and emotion. Also, it provides an overview of pre-existing theories and studies that investigates their relationships.

\section{REFERENCES}

[1] Levinoff, E. J., Li, K. Z., Murtha, S., \& Chertkow, H. (2004). Selective attention impairments in Alzheimer's disease: evidence for dissociable components. Neuropsychology, 18(3), 580.

[2] Fell, J. (2004). Identifying neural correlates of consciousness: The state space approach. Consciousness and Cognition, 13(4), 709-729.

[3] Kranczioch, C., Debener, S., Schwarzbach, J., Goebel, R., \& Engel, A. K. (2005). Neural correlates of conscious perception in the attentional blink. Neuroimage, 24(3), 704-714.

[4] Desimone, R., \& Duncan, J. (1995). Neural mechanisms of selective visual attention. Annual review of neuroscience, 18(1), 193-222.
[5] Hamker, F. H. (2005). The reentry hypothesis: the putative interaction of the frontal eye field, ventrolateral prefrontal cortex, and areas V4, IT for attention and eye movement. Cerebral cortex, 15(4), 431-447.

[6] Vandenberghe, R., Dupont, P., Bruyn, B. D., Bormans, G., Michicls, J., Mortelmans, L., \& Orban, G. A. (1996). The influence of stimulus location on the brain activation pattern in detection and orientation discrimination: A PET study of visual attention. Brain, 119(4), 1263-1276.

[7] Barrett, N. A., Large, M. M., Smith, G. L., Karayanidis, F., Michie, P. T., Kavanagh, D. J. \& O'Sullivan, B. T. (2003). Human brain regions required for the dividing and switching of attention between two features of a single object. Cognitive brain research, 17(1), 1-13.

[8] Pinault, D. (2004). The thalamic reticular nucleus: structure, function and concept. Brain research reviews, 46(1), 1-31.

[9] Lindsley, D. B. (1951). Emotion. In S. S. Stevens (Ed.), Handbook of experimental psychology (p. 473-516). Wiley.

[10] Solomon, R. C. (1993). The philosophy of emotions. Handbook of emotions, 2, 5-13.

[11] Desimone, R. (1998). Visual attention mediated by biased competition in extrastriate visual cortex. Philosophical Transactions of the Royal Society of London. Series B: Biological Sciences, 353(1373), $1245-1255$

[12] Desimone, R., \& Duncan, J. (1995). Neural mechanisms of selective visual attention. Annual review of neuroscience, 18(1), 193-222.

[13] Duncan, J. (2006). EPS Mid-Career Award 2004: brain mechanisms of attention. Quarterly Journal of Experimental Psychology, 59(1), 2-27.

[14] Yiend, J. (2010). The effects of emotion on attention: A review of attentional processing of emotional information. Cognition and emotion, 24(1), 3-47.

[15] Mogg, K., \& Bradley, B. P. (1998). A cognitivemotivational analysis of anxiety. Behaviour research and therapy, 36(9), 809-848.

[16] Yiend, J., Mackintosh, B., \& Savulich, G. (2012). Cognition and emotion.

[17] Kristjánsson, Á., \& Egeth, H. (2020). How feature integration theory integrated cognitive psychology, neurophysiology, and psychophysics. Attention, Perception, \& Psychophysics, 82(1), 7-23. 
[18] Yiend, J. (2010). The effects of emotion on attention: A review of attentional processing of emotional information. Cognition and emotion, 24(1), 3-47.

[19] Kimchi, R. (1988). Selective attention to global and local levels in the comparison of hierarchical patterns. Perception \& Psychophysics, 43(2), 189198.

[20] Fredrickson, B. L., \& Branigan, C. (2005). Positive emotions broaden the scope of attention and thought-action repertoires. Cognition \& emotion, 19(3), 313-332.

[21] Ekman, P., Friesen, W.V., \& Ancoli, S. (1980). Facial signs of emotional experience. Journal of Personality and Social Psychology, 39, 1124-1134.

[22] Forster, J., \& Higgins, E. T. (2005). How global versus local perception fits regulatory focus. Psychological Science, 16, 631-636.

[23] Bradley, M.M., \& Lang, P.J. (1994). Measuring emotion: The Self-Assessment Manikin and the semantic differential. Journal of Behavior Therapy and Experimental Psychiatry, 25, 49-59.

[24] Gable, P. A., \& Harmon-Jones, E. (2008). ApproachMotivated Positive Affect Reduces Breadth of Attention. Psychological Science, 19(5), 476-482.

[25] Schupp, H. T., Flaisch, T., Stockburger, J., \& Junghöfer, M. (2006). Emotion and attention: eventrelated brain potential studies. Progress in brain research, 156, 31-51.

[26] Luck, S.J. and Hillyard, S.A. (2000) The operation of selective attention at multiple stages of processing: evidence from human and monkey electrophysiology. In: Gazzaniga, M.S. (Ed.), The Cognitive Neurosciences (2nd edn). MIT Press, Cambridge.

[27] Stewart, J. L., \& Paulus, M. P. (2013). Neural correlates of craving for psychoactive drugs. Principles of Addiction: Comprehensive Addictive Behaviors and Disorders, 1, 453.

[28] Schupp, H. T., Junghöfer, M., Weike, A. I., \& Hamm, A. O. (2003). Attention and EMOTION: An ERP analysis of facilitated emotional stimulus processing. NeuroReport, 14(8), 1107-1110. 